\title{
The marketing resource management market
}

\section{Chris Bryan}

is a director of consulting with Gartner. He has worked extensively in the areas of MRM, CRM and ERP, helping organisations to make appropriate technology choices and to structure the subsequent implementations to fully exploit the system capabilities and deliver the maximum benefit.

\section{MARKET OVERVIEW}

The marketing resource management (MRM) area continues to be one of the fastest growing sub-segments of the customer relationship management (CRM) segment. However, the dividing line between an MRM and relationship marketing solution is not exact and different vendors draw the line differently. This can be confusing as it becomes difficult to determine which vendors offer appropriate solutions. The MRM sub-segment is extremely active and has drawn the attention of larger vendors. As a result, there has been considerable consolidation which will continue into the future. This paper draws together Gartner's research around the MRM market and the strengths and weaknesses of the players.

Gartner Dataquest reports that the overall worldwide customer relationship management (CRM) market for software licences returned to positive growth during 2004, moving from -4.4 per cent in 2003 to a conservative initial estimate of 2 per cent, with product licence revenue of $\$ 3.45 \mathrm{bn}$, this trend is expected to continue during 2005 , resulting in 2.9 per cent growth. Of the three primary segments, the greatest gains during 2004 occurred in marketing, with more than 31 per cent growth among the top ten vendors. In 2004, the marketing segment represented approximately 15.4 per cent of the total CRM market. Marketing automation applications include marketing resource management, customer management, analytics and partner relationship management (PRM).

Marketing automation is expected to continue to be the fastest growing sub-segment, with an 11.2 per cent compound annual growth rate (CAGR) through 2009, unless the economy worsens significantly, and buyers shift decisions back to solutions focussed on cost optimisation and productivity improvements traditionally associated with customer service and support applications. MRM applications will also continue to gain popularity due to benefits of operational efficiencies and cost reductions. Increased buyer interest in analytics and strong vendor performance in these areas will contribute to growth during the forecast period.

MRM is an emerging category that is attracting a lot of interest from marketing organisations. However, some confusion can arise when elements of MRM are compared with the elements present in most relationship marketing solutions. Categories of functionality (such as collateral management, budgeting and workflow) can be found in both applications, but the role and scope of each is significantly different. 
Relationship marketing solutions tend to operate on a number of assumptions and apply these assumptions to the campaign. MRM solutions tend to focus on establishing the starting premises. For example, that there will be a campaign is assumed by the campaign management system (CMS) tool but planned in the MRM tool. The budget available for the campaign is given to the CMS application but allocated in an MRM application, and the collateral associated with a particular campaign is linked to the campaign by the CMS tool but commissioned, designed, created and tracked by the MRM solution.

Gartner believes that by 2006, fewer than 15 per cent of Global 1000 companies will have enterprise-class MRM deployments. However, between now and 2007, enterprises that successfully deploy MRM solutions will reduce their time to market by more than 40 per cent, realise at least 10 per cent cost savings in their marketing operations and achieve more than 20 per cent higher revenue gains than their peers, giving them a significant competitive advantage. As a consequence, by 2008, MRM capabilities will drive the selection of enterprise marketing applications as competitive pressures lend them greater weight. It is this expectation that has led several analysts to talk about MRM growth as being explosive.

There are approximately 25 vendors operating in the MRM space and Gartner tracks a number of these (see Figure 1). As with any active market, the landscape changes regularly. The major changes since the production of the magic quadrant in Figure 1 have been Oracle's purchase of Siebel during September 2005 and the announcement of the Fusion product line to create a common product from the recent PeopleSoft and JD Edwards acquisitions, and Aprimo's purchase of DoubleClick in
June 2005. Given the predicted growth for this segment, it is likely that there will be continued consolidation.

\section{LARGER COMPANIES}

Gartner regularly publishes research on the largest and most innovative companies in a particular market sector. The following section provides a degree of detail around each of the major players in the MRM sector.

\section{Aprimo}

Aprimo is probably best-known and respected for its MRM capabilities. It continues to execute well in the MRM market and was again the only leader in the 1Q05 MRM Magic Quadrant (Figure 1), based on the breadth and maturity of its MRM offering and its related implementation experience. Aprimo has an extensive consulting arm that approximately equals its software income. Its planning and financial management capabilities continue to be strong differentiators, with growing traction for the create-and-develop functionality. Aprimo's acquisition of Then in July 2004 improved its asset management and marketing fulfillment capabilities and strengthened its vision for MRM analytics. Emphasis in Aprimo 7.0 included enhancements in the user interface, workflow, financials, platform, i-Assets and Aprimo tools. Aprimo is one of the few marketing automation companies to offer event management capability within its suite of solutions for organising and managing industry events.

Aprimo's acquisition of DoubleClick's enterprise marketing solution business unit, following DoubleClick's acquisition of SmartPath in March 2004, gave it improved capabilities in campaign management. However, Aprimo will not have a competitive campaign 


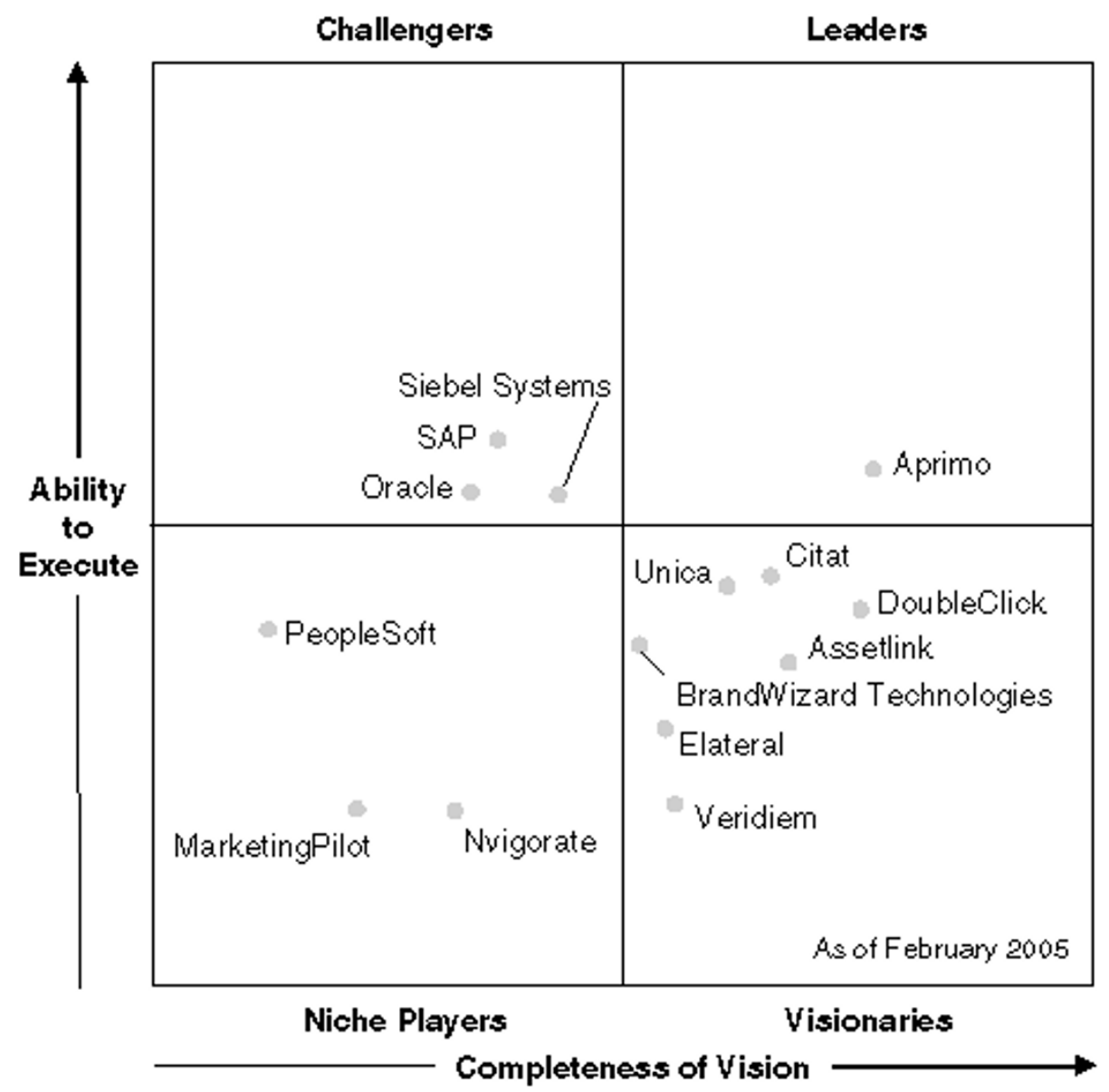

Figure 1: Magic quadrant for marketing resource management, 1Q05

management product until mid-to-late 2007, when it releases Aprimo Marketing 8.0. While this is expected to add key business-to-consumer (B2C)-oriented campaign management functionality, such as a real-time recommendation engine, event triggering, campaign optimisation and relevant areas of e-marketing.

\section{Oracle, PeopleSoft and Siebel}

Oracle remains a challenger because of its strong viability and growing traction for its MRM-related functionality. Oracle's MRM applications leverage core technologies to support project management, content management and marketing fulfilment, so pulling together existing capability within the application suite. Some prospects and customers still cite marketing usability concerns due to reliance on core technologies and the consequent need to have large number of Oracle products, however.

Oracle continues to invest in application usability; Oracle Marketing release v.11.5.10 delivers enhanced ease of use. Performance measurement capabilities will improve with the embedding of daily business intelligence for closed-loop analysis and interactive data mining. Oracle's strength in MRM lies in the tight linkage between MRM, campaign execution and sales force automation (SFA) as well as 
straightforward integration with other Oracle applications and databases.

With the acquisition of PeopleSoft, Oracle gained another set of marketing solutions including its Enterprise CRM product suite and other capabilities within EnterpriseOne. Although it was expected that Oracle would cease to sell the PeopleSoft products, it has, in fact, continued to sell PeopleSoft Enterprise CRM to clients while offering a future product upgrade path through its Project Fusion.

Oracle plans one more major release of its marketing applications for Oracle E-Business Suite 12.0 and PeopleSoft Enterprise 9.0 before its release of the Fusion product set. Although Oracle has made some CRM product decisions around the sales suite, it has not yet made any definitive decisions regarding its set of marketing solutions, beyond this final release. Oracle has committed itself to supporting PeopleSoft and JD Edwards applications on the Oracle Fusion Middleware platform, which will enable PeopleSoft marketing applications to leverage middleware capabilities such as the Oracle Business Process Execution Language (BPEL) for process management, to enable automation of more-complete business processes across marketing and sales, such as lead management. The Fusion roadmap is well publicised, and Oracle is committed to providing a migration path for all of the product sets, however, at the time of writing, there is a lack of detail about what the migration will look like.

Siebel emerged as a challenger with the release of Siebel Marketing Suite 7.7. Its planning, budgeting and production management capabilities are competitive in the market. Siebel offers some marketing fulfilment capabilities for distributors and partners through its Partner Relationship Management solution. Its vision for MRM analytics and closed-loop marketing was one of the strongest in the industry. Siebel offers basic content management capabilities and has partnerships with vendors such as Documentum for more advanced functionality. Customers and prospects have voiced concerns regarding the number of 'clicks' to reach some capabilities; however, Siebel greatly improved overall usability with Siebel 7.7 and its iHelp capability. While this will remain a top tier product in this arena, it is unclear how Oracle will continue to support it. Gartner does not believe that certain elements of Oracle E-Business Suite will be carried forward to supplant Siebel functionality and be the basis of the equivalent parts of the future Product Fusion CRM applications. Oracle products such as Oracle iStore and Oracle Configurator have strong market positions, and Gartner predicts they will become the standard in any new product offering. In areas such as MRM, Oracle and PeopleSoft had stronger content management and workflow than Siebel's application. However, by 2010, at least 80 per cent of Oracle's Project Fusion CRM application functionality will be based on Siebel CRM functionality. Siebel will provide Oracle with a strong and proven CRM product that will form the centrepiece of Oracle's CRM offering pre- and post-Project Fusion.

During the first half of 2006, Oracle will have to make some difficult decisions about reducing investments in the duplicate functionality it has gained from its acquisitions during the past two years. It will need to readjust priorities and focus because of Siebel. This will cause some migration pains for customers during the next few years, as Oracle determines which product components get carried through Oracle Project Fusion. Users should not assume that Oracle will apply the same R\&D effort to all of these products. 


\section{SAP}

SAP, like Oracle, remains a challenger based on its overall viability, large customer base and continuing MRM investments. SAP provides capabilities for planning, budgeting, forecasting and measurement; and portals, along with workflow and content management capabilities, facilitate collaboration for production and knowledge management. With growing customer adoption of SAP's marketing solution, most deployments focus on direct marketing activities for planning, budgeting and executing campaigns and promotional activities. Although SAP has greatly improved its user interface, some prospects, as with Oracle, still cite concerns with marketing usability and flexibility relative to best-of-breed marketing applications.

SAP, by working with its major users, develops capability that has specific vertical market focus. SAP has placed much emphasis on MRM and customer analytics in its recent consumer goods (CG) release. It is gaining momentum in these areas because its products have broad functionality. MRM functionality includes marketing planning, budgeting, forecasting and measurement, workflow, and extended collaboration. SAP is not as developed in business-to-business (B2B) or B2C campaign management as it is in MRMs and it lags the behind best-of-breed vendors. However, based on the emerging requirements of CG companies, basic functionality in campaign planning, development, execution and analytics is sufficient. SAP's marketing limitations be in the lack of integration with multi-channel selling capability, which combines field sales, marketing, national promotions and campaigns in a single offering. SAP, however, provides fully integrated capability between its financial, manufacturing and human resource (HR) capabilities and arguably has the only end-to-end and complete enterprise resource planning and customer relationship management offering available. With Oracle's acquisistion of Siebel and the uncertainty this has created, it is expected that SAP will actively develop detailed functionality in this area and be included in more evaluations.

\section{Unica}

Unica's balance between enabling processes and analytic capability generally appeals to companies specialising in financial services, retail, travel and hospitality and telecommunications essentially, companies with complex requirements for marketing functions, such as cross-campaign optimisation or data mining, or which need integrated sales and marketing or complex marketing resource management. Unica's breadth of marketing functionality is impressive and will not be duplicated by any single vendor (with the possible exception of SAS, through its partnership with Aprimo) before the end of 2006.

Unica offers a suite of applications that enable companies to improve marketing efficiency and effectiveness, particularly in campaign management, regardless of their initial level of sophistication, Unica's strategy in campaign management has been very successful. Although late to the market, it has incorporated strong capabilities to build a broad range of functionality.

In addition to campaign management, Unica's strategy focusses on implementing the marketing suite concept by providing additional capabilities for analytics, MRM and e-marketing. This has enabled Unica to avoid being outflanked by the larger suite vendors and provides differentiation against its more analytically-orientated campaign management competitors. 
The additional software modules enhance Unica's market visibility but do not generate significant revenue independently of CMS. Unica rarely wins stand-alone MRM deals. However, its MRM solution has improved during the past year, particularly with the split of its original Plan module into two modules (Operations Management and Digital Asset Management) and the launch of another module (Financial Management); and called Affinium Plan Operations Management (for strategic planning, project management and marketing workflow) Affinium Plan Digital Asset Management (for content management) and Affinium Plan

Financial Management (for managing the marketing budget).

Unica's strategy has been to provide robust functionality in core areas of the application (such as traditional segmentation and campaign management), while providing a wide range of complementary modules that mimic the directions that other vendors have taken with best-of-breed functionality. Unica offers a broad range of marketing functionality but does not offer the most powerful solution available in areas outside cross-channel campaign management. (For example, Epiphany is stronger for real time, Aprimo is stronger for MRM and SAS is stronger for analytics.) Companies buying from Unica are essentially investing in a wide range of options for improving marketing efficiency and effectiveness.

\section{VISIONARY AND NICHE PLAYERS}

\section{Assetlink}

Assetlink provides a broad vision of MRM and is increasingly competing with Aprimo as a best-of-breed vendor. The Assetlink solution enables marketers to: define their marketing strategy; set goals and metrics; plan; manage tasks, programme execution and assets; manage and track budgets; automate production of artwork; procure and distribute marketing assets; and analyse plans and operations. Assetlink can be considered for a broad range of MRM capabilities, however, smaller vendors invariably confer greater risk for the user, which need to be balanced against robust MRM capabilities and usability.

\section{BrandWizard}

BrandWizard Technologies continues to redefine the brand and production management market for MRM with its visionary focus on create-and-develop functionality. For reporting, it has moved from online transaction processing to online analytical processing capabilities using Microsoft Commerce Server. BrandWizard has established partnerships with Documentum for content management, and with Harte-Hanks and other direct mail/fulfillment companies in the Omnicom Group, for fulfillment and distribution. During 2004, it doubled its customer base from 20 to 40 customers. BrandWizard is part of Interbrand, an Omnicom company, giving it access to a large sales distribution network. BrandWizard should be considered by organisations interested in a best-of-breed MRM solution for creating and developing marketing content.

\section{Citat}

Citat's European installed base revenue grew by 60 per cent in 2004 . It integrated its numerous 2003 acquisitions, including Traffic, into MarketStore Citat's MRM solution. New features include a content creation engine, a workflow editor, project management 
capabilities for production control, a module for product information, enhanced digital asset management capabilities and search functionality. Citat also integrates with Microsoft Content Management Server. During 2004, Citat launched Citat Client Community (3C) for MarketStore users. European companies interested in brand control and production management should consider Citat.

\section{Elateral}

Elateral provides a strong vision for a tightly integrated approach to content creation and customisation, asset/content management and marketing fulfilment, which includes integration with creative agencies and a highly scalable architecture for large distributed sales and distribution networks. Although

customers say that the solution is flexible, other references have cited issues with usability in document design/creation, where design users require practice and training to leverage advanced capabilities effectively. Elateral continues to expand its penetration in its existing customers, but new customer growth is slow.

\section{Veridiem}

Veridiem retains its visionary focus on MRM analytics for data gathering and analysis to drive marketing effectiveness. During 2Q04, it successfully closed a new round of funding and launched Veridiem 2. The company continues to focus primarily on consumer goods and automotive industries. Although most MRM vendors support a top-down approach to planning and budgeting, Veridiem offers a bottom-up approach. Using advanced analysis and marketing mix optimisation, the Veridiem 2 analytical platform helps customers allocate their marketing budgets more effectively by product, media and metrics (that is, sales, traffic, brand, time period and so on). For consumer packaged goods and automotive companies, Veridiem complements other MRM applications that focus on operational execution.

\section{MarketingPilot}

MarketingPilot offers a functionally rich, low-cost MRM application based on client/server architecture to the midmarket. It planned to release a .NET thin-client solution during 2005.

Movement to the .NET platform should improve penetration in the

larger-enterprise market, improving its position on the MRM Magic Quadrant. It will need stronger partnerships with resellers, marketing consultancies and systems integrators. With the release of MarketingPilot R6.5, the company offers several new features for event and trade show management, project request management, approval management and improved tracking of jobs, tasks and events. Midsize businesses with less than 50 'marketing' users should consider MarketingPilot.

\section{Nvigorate}

Nvigorate offers a thin-client solution for MRM. Oneplace enables companies to: manage their brand and digital assets, the creative development process, marketing programmes and budgets; customise advertising templates and to track spending. Although Nvigorate has a growing customer base, it has few live production clients to date. Nvigorate must gain broader market momentum and solidify strong sales and distribution partnerships to prove the value of its consultancy-driven approach. It has partly addressed this by acquiring the marketing software division, associated customers 
and staff from Sygnet Interactive. Sygnet has strong capabilities in collateral and advertising creation, fulfilment and delivery, as well as expertise in content management for marketing. Nvigorate can be considered as an alternative to the other best-of-breed MRM vendors, but, as before, one must balance risk trade-offs with having to do business with a small vendor.

\section{FUTURE OF MRM}

The predicted competitive advantage conferred through the deployment of MRM techniques has not only made this the fastest growing relationship management segment but has also drawn it to the attention of the major enterprise application vendors. In most organisations, the marketing function has to balance its creative approach against process driven management and so has an increasing need to measure and justify its activities. The developments in the above products follow this trend, by providing not just improved capability and functional integration but also improved screen design and workflow.
There will continue to be increased consolidation in the market, with vendors buying in missing capability in preference to developing it from scratch in order to shorten the time-to-market. There is a balance to be struck here, however, between having too big a mouthful to swallow and integrating the products effectively to quickly add market share.

Driven by the perceived growth many companies with a background in CRM and marketing content are likely to build products to enter this segment. These are likely to include pure CRM vendors such as Chordiant and SAS, as well as digital asset management companies such as Interwoven and Documentum. The financial management aspects of MRM may also prompt vendors such as Sage and Sun Systems to form alliances with marketing vendors to address non-corporate departmental user's needs. Users will need to balance the short term return on investment requirements of a point solution against the broader integrated needs of the wider organisation and adopt approaches that ensure maximum return. 